
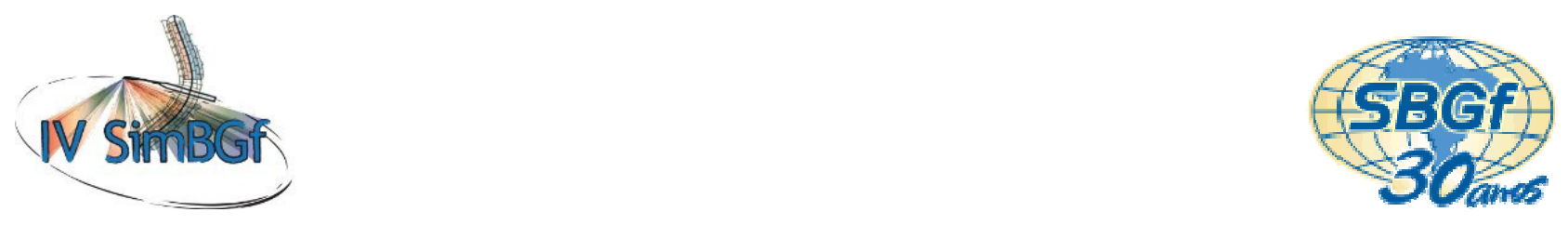

\title{
Um Modelo Matemático para Filtragem de Perfis de Poço utilizando Parâmetros Fuzzy Diferenciais
}

\author{
Carlos E. Guerra, UFPA/CPGF, Santarém/Belém, Brasil \\ Ivnon J. Farias Lima, UFPA/CPGF, Belém, Brasil \\ Copyright 2009, SBGf - Sociedade Brasileira de Geofísica \\ This paper was prepared for presentation during the $11^{\text {th }}$ International Congress of the \\ Brazilian Geophysical Society held in Salvador, Brazil, August 24-28, 2009. \\ Contents of this paper were reviewed by the Technical Committee of the $11^{\text {th }}$ \\ International Congress of the Brazilian Geophysical Society and do not necessarily \\ represent any position of the SBGf, its officers or members. Electronic reproduction or \\ of the Brazilian Geophysical Society is prohibited.
}

\begin{abstract}
We can characterize the signal processing as a set of techniques used to extract information from a signal. A signal can be described mathematically as a function of several variables (parameters), for example, time, distance, resistivity, radiance, among and so forth. The signal acquisition is usually made by one or more devices electric / electronic (analog and / or digital), as examples, a temperature sensor, a digital camera, a probe for resistivity, and so forth. From this point, the type of processing to be applied will depend on the nature of the signal and its information. During the acquisition process, the recorded signal may contain, in addition to the information desired, also a number of spurious information (no connection or interest) called noise. The presence of noise in the signals can impair the process of extracting the desired information, leading to ambiguous results and no link. Thus the goal of this work is to show a non-linear technique for noise removal (fuzzy logic and sets) and apply it to well logs.
\end{abstract}

\section{Resumo}

Podemos caracterizar o processamento de sinais como um conjunto de técnicas utilizadas para extrair informações de um sinal qualquer. Um sinal pode ser caracterizado matematicamente como sendo uma função de várias variáveis (parâmetros), como exemplo, tempo, distância, resistividade, radiância, dentre outros. $A$ aquisição de um sinal é normalmente feita por um ou mais dispositivos eletro/eletrônicos (analógicos e/ou digitais), como exemplos, um sensor de temperatura, uma câmera digital, uma sonda de resistividade, dentre outros. A partir deste ponto, o tipo de processamento a ser aplicado dependerá da natureza do sinal e da sua informação. Durante o processo de aquisição, o sinal registrado poderá conter, além da informação desejada, também uma quantidade de informação espúria (sem nexo ou interesse) denominada por ruído. A presença de ruídos nos sinais pode prejudicar os processos de extração da informação desejada, levando a resultados ambíguos e sem nexo. Assim o objetivo deste trabalho é mostras uma técnica não linear para a extração de ruídos em perfis de poços utilizando à lógica FUZZY.

\section{Introdução}

O Processamento de Sinais consiste na análise e/ou modificação de sinais de forma a extrair informações dos mesmos e/ou torná-los mais apropriados para alguma aplicação específica. Este processamento pode ser feito de forma analógica ou digital. As técnicas de processamento de sinais podem ser de muita utilidade no controle e análise de sistemas físicos de interesse das mais diversas áreas, engenharia, economia, biologia, geociência, saúde, etc. A filtragem é uma das aplicações típicas do processamento de sinais, neste contexto, entende-se por filtragem a remoção de ruído visando melhorias na interpretação e utilização dos dados. Neste trabalho, abordaremos o processo de filtragem digital baseada na lógica fuzzy aplicada em dados sintéticos e reais de perfis de poço. Perfil de Poço é a forma de apresentação das variações das propriedades físicas mensuradas ao longo da profundidade de um poço. Originalmente, as medidas eram tomadas na forma analógica e o perfil apresentado na forma gráfica, diretamente no papel. Atualmente, as medidas são registradas na forma digital e armazenadas na forma de arquivos do tipo ASCII, como normatizado pela API (American Petroleum Institute). Estas medidas vem acrescidas por níveis de ruídos, devido à calibração das sondas, problemas mecânicos, speckle, interferências, dentre outros.

Para minimizar os efeitos dos ruídos nos perfis, existem algumas técnicas básicas, baseadas na análise Fourier e procedimentos de inversão, que conseguem obter alguns resultados (GUERRA, C. E., 1994). Entretanto, limitações de ordem tecnológicas (obtenção das funções respostas das ferramentas) e problemas numéricos (obtenção e uso das funções de transferências para os filtros, erros computacionais, estimativas da razão sinal-ruído,...) constituem problemas que nem sempre podem ser contornados satisfatoriamente. Desta forma, os algoritmos cognitivos, dentre os quais se encaixam os sistemas FUZZY, oferecem novas alternativas para o tratamento de dados que envolvam incertezas (Miranda, 1998; Russo \& Ramponi, 1996) e ambigüidades (os perfis de poços, por exemplo) .

Os sistemas FUZZY baseiam-se em operações que envolve conjuntos e lógica nebulosa (Aguiar \& Oliveira, 
1999). A lógica fuzzy, por exemplo, difere da lógica clássica, no que se refere ao mapeamento dos valores "verdade" e "falso". Na teoria clássica dos conjuntos, se um elemento pertence a um conjunto ou não, i. é, o fator de pertinência pode assumir somente valores 0 ou 1 . 0 valor 0 indica uma completa exclusão e um valor 1 representa uma completa pertinência. Logo, dado um universo $U$ e um elemento particular $x \notin U$, a função de pertinência $\mu_{\mathrm{A}}(x)$ com respeito a um conjunto $A \subseteq U$, é dada por (Barron, 1993):

$$
\mu_{A}(x)=\left\{\begin{array}{lll}
1 & \text { se } x & \in A \\
0 & \text { se } x & \notin A
\end{array}\right.
$$

Por outro lado, um conjunto fuzzy A, definido no universo de discurso $X$ é caracterizado por uma função de pertinência $\mu_{A}$, a qual mapeia os elementos de $X$ para 0 intervalo $[0,1]$ :

$$
\boldsymbol{\mu}_{\mathrm{A}(\mathrm{X})} \rightarrow[\mathbf{0 , 1}]
$$

Desta forma, a função de pertinência associa a cada elemento $x$ pertencente a $X$ um número real $\mu_{\mathrm{A}(\mathrm{X})}$ no intervalo $[0,1]$, que representa o grau de pertinência do elemento $x$ ao conjunto $A$, isto é, o quanto é possível para o elemento $x$ pertencer ao conjunto $A$ :

$$
\begin{gathered}
\boldsymbol{\mu}_{\mathbf{A}(\mathbf{X})}=\mathbf{X} \rightarrow[\mathbf{0}, \mathbf{1}], \boldsymbol{\mu}_{\mathbf{A}(\mathbf{X})}=\mathbf{0} \\
\mathbf{0}<\boldsymbol{\mu}_{\mathbf{A}(\mathbf{X})}<1 \\
\boldsymbol{\mu}_{\mathbf{A}(\mathbf{X})}=\mathbf{1} \\
\mathbf{A}=\left\{\left(\mathbf{X}, \boldsymbol{\mu}_{\mathbf{A}}(\mathbf{x})\right) / \mathbf{X} \in \mathbf{X}\right\}
\end{gathered}
$$

Finalmente, a lógica fuzzy busca uma generalização da lógica clássica, flexibilizando-a para assumir valores no intervalo $[0,1]$.

\section{Metodologia}

Os sistemas fuzzy's foram projetados para trabalharem com dados que carregam um certo grau de incertezas, imprecisões e/ou ambiguidades. A presença de ruídos nas medidas de perfilagem aumenta ainda mais estas incertezas. Diante destas características citadas anteriormente, foi desenvolvido um um modelo matemático baseado na lógica Fuzzy, para a remoção dos ruídos presentes em perfis de poços. Em cada ponto do perfil, calculamos um diferencial à esquerda e outro à direita. A continuidade do sinal é então avaliada testandose os sinais dos diferenciais, através das regras de inferências. Diferenciais com sinais opostos indicam continuidade do perfil, enquanto que diferenciais com mesmo sinal indicam um salto no ponto considerado. Como resultado do processo de defuzzyficação gera-se um fator de correção de forma a eliminar ou atenuar este salto. Na figura (1) é mostrado o diagrama de blocos simplificado desse modelo de aplicação do filtro fuzzy diferencial.

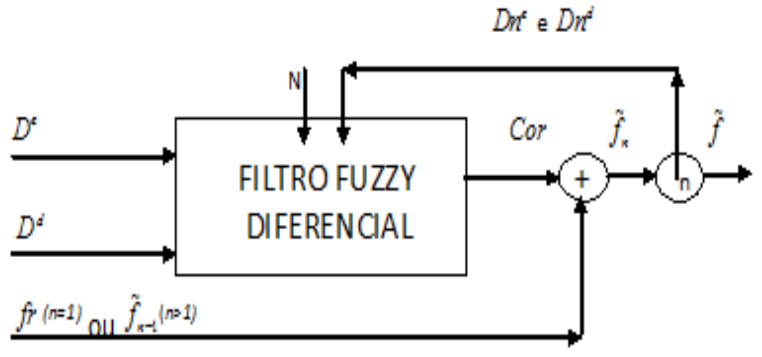

Figura (1): Diagrama do filtro fuzzy diferencial

Onde $f r$ são as medidas do perfil ruidoso, Cor são os fatores de correções chalculados pelo filtro, $D n^{e} e D n^{d}$ são os parâmetros diferenciais na n-ésima interação, $\tilde{f}_{n}$ são as medidas filtradas na n-ésima interação e $\tilde{f}$ são as medidas finais após $\mathrm{N}$ iterações.

O modelo matemático do Sistema Fuzzy, aplicado neste trabalho é composta pelas seguintes etapas:

1- Definição das Variáveis de Entrada:Como variáveis de entrada, adotou-se os diferenciais de propriedades petrofísicas do perfil de poço.

- Parâmetros diferencias iniciais (primeira interação):

$$
\begin{aligned}
& D_{i}^{e}\left(z_{i}\right) \equiv f r_{i-1}\left(z_{i-1}\right)-f r_{i}\left(z_{i}\right) \\
& D_{i}^{d}\left(z_{i}\right) \equiv f r_{i+1}\left(z_{i+1}\right)-f r_{i}\left(z_{i}\right)
\end{aligned}
$$

- Parâmetros diferencias iniciais na n-ésima interação:

$$
\begin{aligned}
& \boldsymbol{D} \boldsymbol{n}_{\boldsymbol{i}}^{\boldsymbol{e}}\left(\mathbf{z}_{\boldsymbol{i}}\right) \equiv \tilde{f}_{n, i-1}\left(\mathbf{z}_{\boldsymbol{i}-\mathbf{1}}\right)-\tilde{f}_{n, i}\left(\mathbf{z}_{\boldsymbol{i}}\right) \\
& \boldsymbol{D n}_{\boldsymbol{i}}^{\boldsymbol{d}}\left(\mathbf{z}_{\boldsymbol{i}}\right) \equiv \tilde{f}_{n, i+1}\left(\mathbf{z}_{\boldsymbol{i}+\mathbf{1}}\right)-\tilde{f}_{n, i}\left(\mathbf{z}_{\boldsymbol{i}}\right)
\end{aligned}
$$

Onde: $D_{i}^{e}\left(z_{i}\right)$ e $D n_{i}^{e}\left(z_{i}\right)$ são os parâmetros diferenciais a esquerda e $D_{i}^{d}\left(z_{i}\right)$ e $D n_{i}^{d}\left(z_{i}\right)$, os parâmetros diferenciais a direita, em cada profundidade $z_{i}$, com um certo número de interações $n$.

2-Inicialização dos parâmetros para o filtro fuzzy: Nesta etapa o filtro é inicializado quanto à estimativa inicial do nível de ruído do perfil (universo de discurso) e mais o número de iterações que será necessário para a filtragem dos dados.

O universo de discurso é definido como:

$$
X n=[-n r, n r]
$$

onde $n r$ é uma estimativa do máximo nível de ruído em módulo e é dado como:

$$
n r=\max \left(\max \left(D^{e+}\right), \max \left(D^{d+}\right)\right)
$$


3- Fuzzificação: é a etapa no qual procede-se o mapeamento do conjunto "crisp" (os parâmetros diferencias a esquerda e a direita) em um ou mais conjuntos no intervalo $[0,1]$, esses conjuntos são as variáveis linguísticas, que representam os nomes associados a cada tipo de parâmetro diferencial. Esta etapa, engloba também, definição das funções de pertinência. Na definição das funções de pertinência para as variáveis, foi utilizada a Função "Gaussiana (ORTEGA, 2001; Gomide et al, 1994) "cujo modelo matemático é dado por:

$$
\operatorname{gaussm} f(x ; c, \sigma)=e^{-\frac{1}{2}\left(\frac{x-c}{\sigma}\right)^{2}}
$$

Em que $c$ é a media e $\sigma$ é o desvio padrão.

A partir dessa função de pertinência, são definidos os conjuntos fuzzy diferenciais à esquerda e a direita como :

$$
\begin{aligned}
& F D^{T I}=\left\{\left(x, \mu_{F D^{T I}}(x)\right)\right\}, x \in X n \\
& e \mu_{F D^{T I}}: X n \rightarrow[0,1] \operatorname{com} \mu_{F D^{T I}}(x)=e^{\frac{-\left(x-c_{T I}\right)^{2}}{2 \sigma_{T I}^{2}}} \\
& T I=\{e-, e+, d-, d+\}
\end{aligned}
$$

Onde $F D^{T I}$ é uma família de conjuntos fuzzy diferenciais cujos sobre-escritos TI indicam a natureza de cada conjunto: diferencial a esquerda negativo ou positivo e diferencial à direita negativo ou positivo. $\mu_{F D^{T I}}$, são funções de pertinências do tipo gaussianas, $\operatorname{com} c_{T I}$ e $\sigma_{T I}$ representando a média e o desvio padrão das medidas dentro do universo de discurso. Na Figura (2), temos um exemplo de como é projetada as funções de pertinências para os parâmetros diferenciais a direita, positivo e negativo. Os parâmetros $c_{T I}$ e $\sigma_{T I}$ dão forma as curvas gaussianas, informando o quão negativo ou positivo é o parâmetro diferencial.

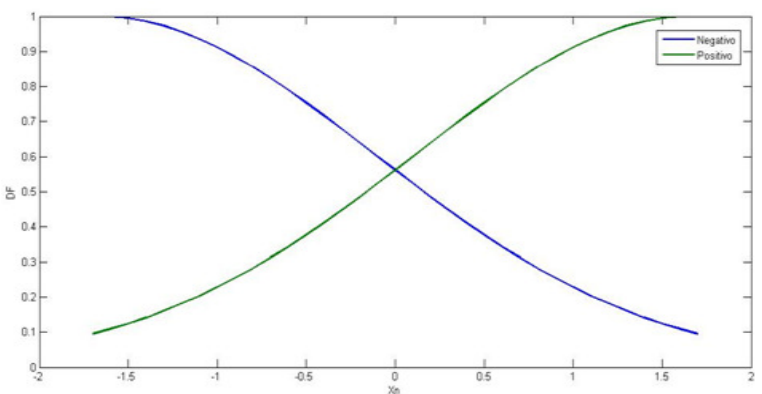

Figura (2): Para o parâmetro diferencial á direita negativo temos $c_{d-}=1.55$ e $\sigma_{d-}=-1.67$ enquanto que para 0 parâmetro diferencial à direita positivo $c_{d+}=-1.55$ e $\sigma_{d+}=1.67$.

Com isto, temos as variáveis lingüísticas. Neste caso temos que cada um dos nomes abaixo representa um conjunto fuzzy na forma:

$$
\begin{aligned}
& \text { positivo }^{e}=F D^{e+}\left(D^{e}\right), \\
& \text { negativo }^{e}=F D^{e-}\left(D^{e}\right), \\
& \text { positivo }^{d}=F D^{d+}\left(D^{d}\right), \\
& \text { negativo }^{d}=F D^{d-}\left(D^{d}\right) .
\end{aligned}
$$

4- Processso de inferência fuzzy: etapa a qual consiste de dois passos: avaliação da premissa de cada regra (conjunção), através dos operadores t-norma (mínimo), e em seguida a etapa de agregação, ponderando as diferentes conclusões das regras ativas sob o operador snorma (Maximo). Os operadores de t-norma e s-norma são normas e co-normas triangulares que fornecerão os métodos genéricos para as operações de intersecção e união nos conjuntos fuzzy, formados na etapa anterior (Fuzzificação). As regras de implicação utilizadas é do tipo Mandani . No modelo foram utilizadas duas regras:

$\checkmark \quad \mathbf{R} 1$ : "Se $D_{i}^{e}\left(z_{i}\right)$ e $D_{i}^{d}\left(z_{i}\right)$ for positivo, então Cor $_{i}$ será positivo;

$\checkmark \quad \mathbf{R 2}$ : "Se $D_{i}^{e}\left(z_{i}\right)$ e $D_{i}^{d}\left(z_{i}\right)$ for negativo, então $\mathrm{Cor}_{i}$ será negativo;

O termo $\otimes \min \left(a^{+} \wedge b^{+}, \operatorname{Cor}^{+}\right)$

$\otimes \min \left(a^{-} \wedge b^{-}, \operatorname{Cor}^{-}\right)$são as regras de implicações Mandami, que nada mais é do o conjunto de funções de pertinências formado por $\left\{\min \left(a_{i}^{+} \wedge b_{i}^{+}, \operatorname{Cor}^{+}\right)\right\} \mathrm{e}$ $\left\{\min \left(a_{\grave{i}}^{-} \wedge b_{i}^{-}, \operatorname{Cor}^{-}\right)\right\}$onde $i$ representa o $i$-ésimo ponto ou amostra considerada. Tomando-se elemento a elemento, i.é, para cada profundidade $\mathrm{z}_{\mathrm{i}}$, as operações acima são efetuadas com base na norma-T (figura 3 e 4):

$$
\begin{aligned}
& \mathrm{R} 1: \mu_{F D^{e+}}\left(D_{i}^{e}\right) \wedge \mu_{F D^{d+}}\left(D_{i}^{d}\right) \Rightarrow \operatorname{Cor}^{+} \equiv \\
& \equiv \min \left(\min \left(\mu_{F D^{e+}}\left(D_{i}^{e}\right), \mu_{F D^{d+}}\left(D_{i}^{d}\right)\right), \operatorname{Cor}^{+}\right) \\
& \operatorname{Cor}^{+}=\mu_{C O R^{+}}(C O R)
\end{aligned}
$$
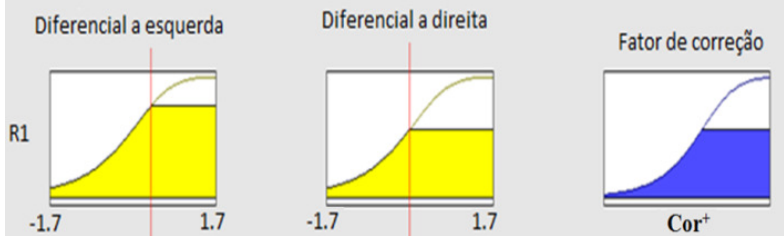

Figura 3: Regra (1) de implicações Mandami, (norma-T ou operador de mínimo).

$$
\begin{aligned}
& \mathrm{R} 2: \mu_{F D^{e^{-}}}\left(D_{i}^{e}\right) \wedge \mu_{F D^{d-}}\left(D_{i}^{d}\right) \Rightarrow \operatorname{Cor}^{-} \equiv \\
& \equiv \min \left(\min \left(\mu_{F D^{e-}}\left(D_{i}^{e}\right), \mu_{F D^{d+}}\left(D_{i}^{d}\right)\right), \operatorname{Cor}^{-}\right) \\
& \operatorname{Cor}^{-}=\mu_{C O R^{-}}(C O R)
\end{aligned}
$$
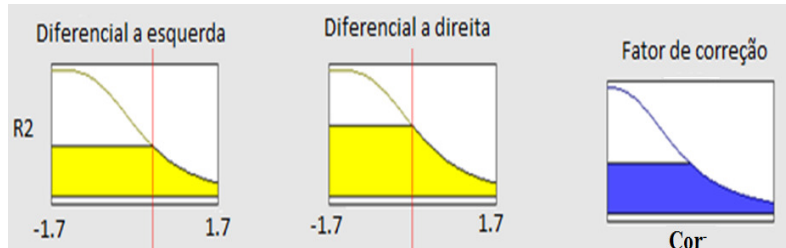

Figura 4: Regra (2) de implicações Mandami, (norma-T ou operador de mínimo). 
Temos como resultado do processo de inferência uma sequência de conjuntos fuzzy relativos a aplicação de cada umas das regras. Para proceder à próxima etapa da defuzzificação é necessário agregar estes conjuntos em apenas um conjunto, através de um operador de agregação, utilizando a conorma-T (operador de máximo) figura (5):

$$
h_{i}(C O R)=\max \left(\operatorname{Cor}_{i}^{+}, \operatorname{Cor}_{i}^{-}\right)
$$

onde $i$ representa cada amostra considerada e $h_{i}(C O R)$ é uma função de pertinência, i.é, $h_{i}: X n \rightarrow[0,1]$

Dado o universo de discurso Xn (Valores de correção), definimos os conjuntos fuzzy corretivos de saída como sendo:

$$
\begin{aligned}
& \operatorname{Cor}^{I I}=\left\{\left(C O R, \mu_{C O R^{I I}}(C O R)\right)\right\}, C O R \in X n \\
& \text { e } \mu_{C O R^{I I}}: X n \rightarrow[0,1] \operatorname{com} \mu_{C O R^{I I}}(C O R)=e^{\frac{-\left(C O R-c_{I I}\right)^{2}}{2 \sigma_{T I}^{2}}}, \\
& T I=\{-,+\}
\end{aligned}
$$

onde $\operatorname{Cor}^{T I}$ é uma família de conjuntos fuzzy corretivos, cujos sobre-escritos TI indicam a natureza de cada conjunto: correção subtrativa e aditiva. $\quad \mu_{C O R^{T I}}(C O R)$ são funções de pertinências do tipo gaussianas, com $c_{T I}$ e $\sigma_{T I}$ representando a média e o desvio padrão das medidas dentro do universo de discurso.

\section{Figura(5): Processo de} agregação dos fatores de orreção pelo operador de agregação, conorma- $T$ (operador de máximo).

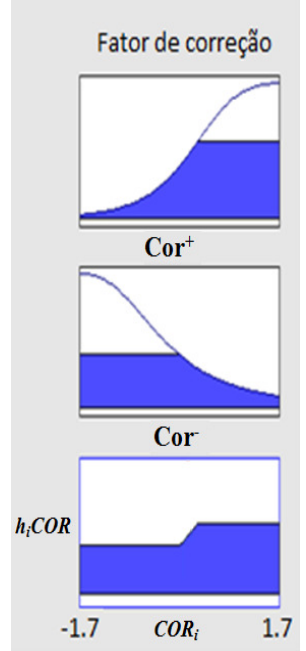

5- Processo de Defuzzyficação: ocorre após o processo de agregação. É nesta etapa que é gerado do fator de correção; (positivo) se o ruído for subtrativo e (negativo) se o ruído for aditivo. A defuzzyficação tem como entrada os valores da função de pertinência $h_{i}(C O R)$. O método de defuzzyficação adotado é o do centróide, onde $n$ é o número de pontos de $h_{j}(C O R)$ :

$$
\operatorname{Cor}_{j}=\frac{\sum_{i=1}^{n} \operatorname{COR}_{i} h_{j}\left(C O R_{i}\right)}{\sum_{i=1}^{n} h_{j}\left(C O R_{i}\right)}
$$

Cor $_{j}$ representa a correção a ser somada na $j$-ésima medida do perfil, na profundidade $z_{j}$ O universo de discursos dos conjuntos fuzzy de saída é o mesmo dos conjuntos fuzzy diferenciais, uma vez que os valores de correção estão dentro do intervalo de níveis de ruídos estimados, figura (6).

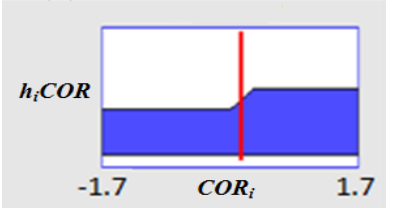

Figura 6: Processo de defuzzyficação.

Na figura (7), podemos ver com mais clareza o Sistema de Inferência Fuzzy aplicado junto com suas regra e o processo de defuzzyficação.

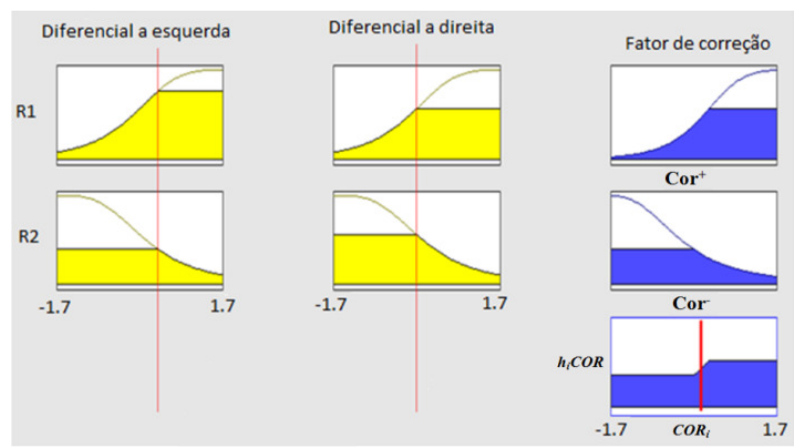

Figura (7):Sistema de inferência fuzzy e defuzzyficação.

6- Correção do Perfil: é o processo que implementa o fator de correção no perfil, extraindo ou atenuando o ruído do sinal, melhorando assim, a qualidade e interpretação dos dados. O fator de correção pode ser aditivo ou subtrativo dependendo do efeito do ruído no perfil. O valor do perfil é corrigido somando-se o valor obtido na defuzzyficação com a medida do perfil ruidoso, i.é, para cada $n$-ésima iteração:

$$
\begin{aligned}
& \tilde{f}_{n}=f_{n-1}+\operatorname{Cor}, n>1 ; \\
& \tilde{f}_{n}=f r+\operatorname{Cor}, n=1 ;
\end{aligned}
$$

Isto significa que para cada medida $f r_{i}$ na profundidade $z_{j}$ temos:

$$
\begin{aligned}
& \tilde{f}_{n, i}\left(z_{i}\right)=\operatorname{fr}\left(z_{i}\right)_{i}+\operatorname{Cor}_{i}\left(z_{i}\right), n=1, \\
& \tilde{f}_{n, i}\left(z_{i}\right)=f_{n-1, i}\left(z_{i}\right)_{i}+\operatorname{Cor}_{i}\left(z_{i}\right), n>1,
\end{aligned}
$$

7- Calculo dos novos parâmetros de entrada do filtro para uma nova iteração: Nesta etapa o perfil filtrado é avaliado quanto aos níveis de correção aplicados. Este procedimento é necessário para a re-calibração do sistema FUZZY e para a execução de uma nova iteração.

Para as interações subsequentes, o universo de discurso e as funções de pertinências são atualizadas, de forma a 
produzir um nível de filtragem menor, baseado em uma nova estimativa, utilizando-se $\tilde{f} n$ e $n r$ :

$$
\begin{aligned}
& n r=\max \left(\max \left(D n^{e+}\right), \max \left(D n^{d+}\right)\right) \\
& D n_{i}^{e}\left(z_{i}\right) \equiv \tilde{f}_{n, i-1}\left(z_{i-1}\right)-\tilde{f}_{n, i}\left(z_{i}\right) \\
& D n_{i}^{d}\left(z_{i}\right) \equiv \tilde{f}_{n, i+1}\left(z_{i+1}\right)-\tilde{f}_{n, i}\left(z_{i}\right)
\end{aligned}
$$

O Processo se repete até a última interação onde então teremos o resultado final do processo de filtragem:

$$
\tilde{f}=\tilde{f}_{n}, n=N
$$

Como pode ser observado, o modelo matemático deste filtro é extremamente simples e baseia-se na suposição de que os ruídos podem ser aditivos (aumenta o nível do sinal) ou subtrativos (diminui o nível do sinal), desta forma, os ruídos são encarados como descontinuidades no sinal. Os parâmetros diferenciais de entrada funcionam como um "detector" para estas descontinuidades, e também como um estimador do nível de ruído. $O$ algoritmo deste filtro permite 0 estabelecimento do nível de filtragem, através do número de iterações. .

\section{Análise dos Resultados}

O processo de filtragem foi aplicado em perfis sintéticos de forma a obter a extração de ruídos presentes nestes sinais. Para os dados sintéticos, foram gerados perfis de Indução baseado no fator geométrico $\boldsymbol{g}$ de DOLL (Doll1949; Serra, 1984; Ellis, 1987), simulando uma ferramenta com duas bobinas, com afastamento $\mathbf{L}$ igual, taxa de amostragem de $\mathbf{0 . 2 m}$ e profundidade inicial $\boldsymbol{z i}$ igual a100m. Como modelo de distribuição de condutividades, adotou-se uma função contínua por partes, onde o domínio representa as profundidades e os intervalos imagens representam as camadas com os respectivos valores de condutividades. A seguir o perfil de condutividade resultante foi contaminado por ruídos gaussianos como é mostrado na Figura 8 , em que adicionamos um ruído positivo e outro negativo os quais são destacados pelos parâmetros diferencias a esquerda e a direita, sendo assim possível verificar a validade do método e sua eficácia. Na figura 9 tem-se o perfil filtrado pelo sistema fuzzy proposto.

Para mostrar a confiabilidade da metodologia foi feita uma comparação entre 0 método de filtragem desenvolvido com lógica fuzzy e o método de filtragem clássica de mediana, sendo o método mais utilizado no processamento de sinais (Russo \& Ramponi, 1996). Na Figura (10), temos um outro exemplo de perfil tratado tratado com o filtro fuzzy, com o numero de interações $\mathrm{N}=2$. Porém, aplicou-se também o filtro clássico de mediana com dois pontos. Como resultado, podemos observar que o filtro fuzzy produziu uma significativa redução dos níveis de ruído em comparação ao filtro de mediana (figura 11). Como no método proposto cada iteração significa um procedimento de filtragem, o filtro fuzzy permite aumentar e diminuir sempre que necessário o nível de filtragem para obter a filtragem correta nos dados considerados.

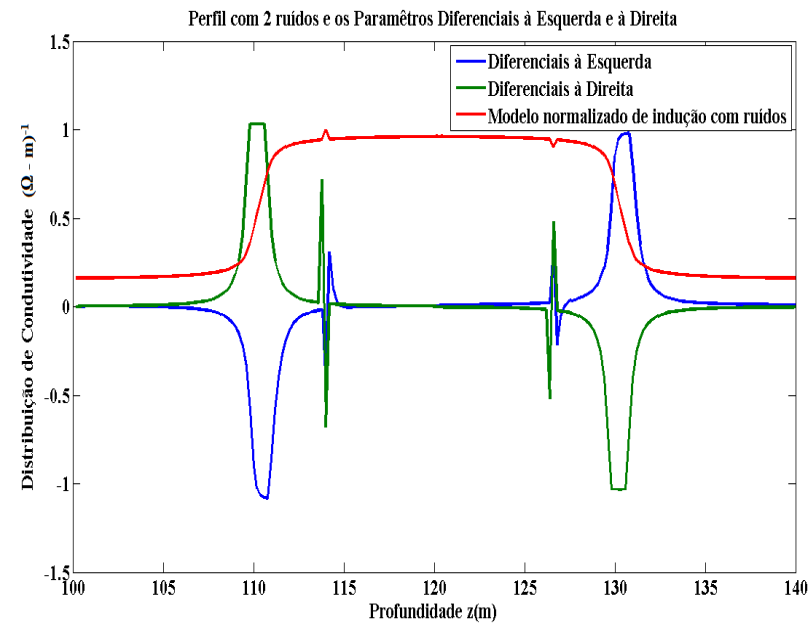

Figura 8: Modelo normalizado com ruídos destacados pelos parâmetros diferencias, a esquerda e a direita.

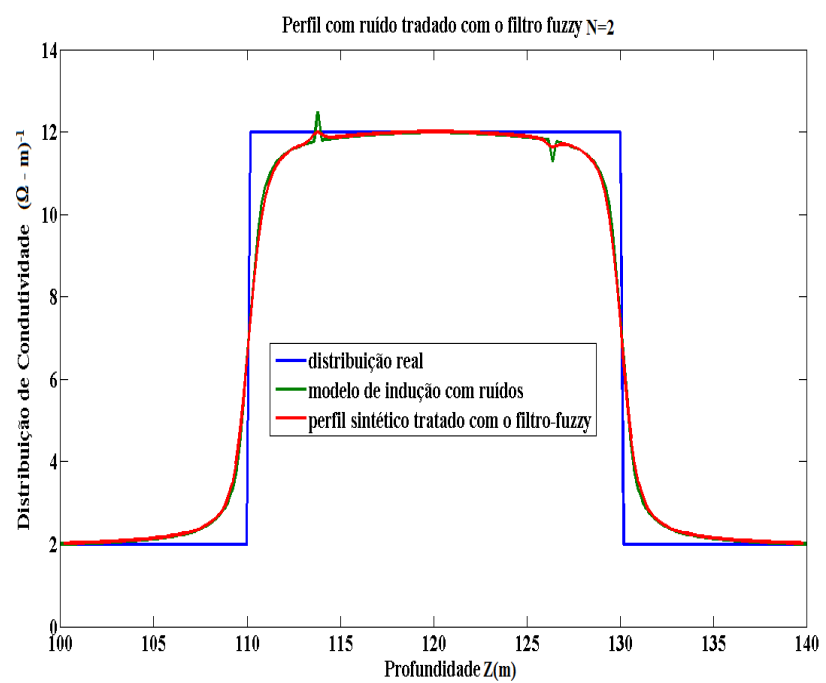

Figura 9: Mostra o perfil sintético corrigido com o filtro fuzzy com numero de interações igual a dois $(n=2)$.

Para mostramos com mais clareza a eficácia do filtro fuzzy em comparação com o filtro de mediana, na Figura (11), é mostrado um trecho destacado do perfil filtrado (Fig. 10). Podemos observar e comprovar que o filtro fuzzy, com duas iterações, foi muito mais efetivo no que diz respeito às atenuações das oscilações provocadas pelos ruídos, suavizando os picos das curvas em todo o trecho, enquanto que o filtro de mediana com dois pontos não foi capaz de remover estas oscilações, diminuindo apenas os picos em algumas partes do perfil. 


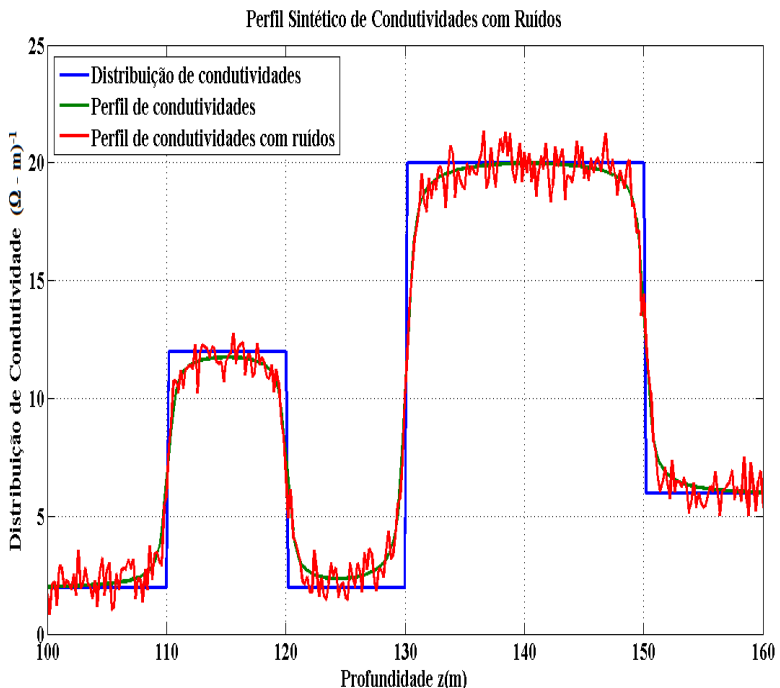

Figura 10: Perfil de condutividade sintético, sem ruído (verde), com ruído (vermelho) e a distribuição de condutividades (em azul).

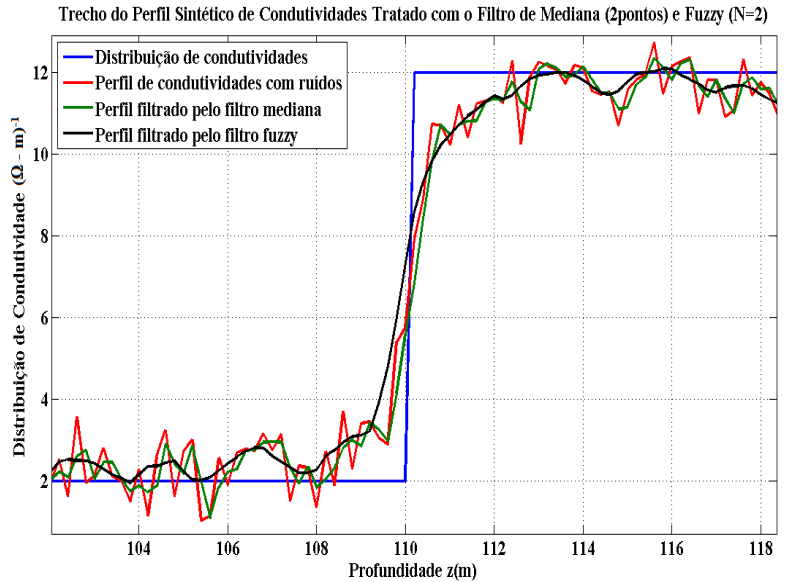

Figura 11: trecho do Perfil de condutividade tratado com o filtro de mediana (2 pontos) e o filtro fuzzy com 2 iterações $(\mathrm{N}=2)$, em que temos a distribuição de condutividades (azul), perfil de condutividade com ruído (vermelho), perfil filtrado com o filtro de mediana (verde) e perfil filtrado com filtro fuzzy (preto).

\section{Conclusões}

A metodologia funcionou satisfatoriamente bem quando aplicada em dados sintéticos e reais, produzindo uma atenuação significativa dos níveis de ruídos. Foram necessárias apenas duas iterações para se chegar a um bom resultado, o que mostra a convergência do algoritmo. Nota-se que para os dados Sintéticos, a escolha do número de iterações do processo é fundamental no sentido de diminuir o nível de ruídos e manter a integridade do sinal, i é, a informação litológica. Outro ponto importante da metodologia é o fato de que o processo de filtragem não produz deslocamentos no perfil filtrado. Já a definição dos parâmetros diferenciais como entrada do sistema fuzzy e como objetos de inferência, foi importante no sentido de avaliar a continuidade do sinal-perfil e os níveis de ruído; evitando filtragens desnecessárias e perda de sinal. Em trabalhos futuros uma melhor definição dos parâmetros de entrada, de inferências, das funções de pertinências e de critérios para a escolha do número de iterações serão necessários para a simplificação e otimização do algoritmo de filtragem fuzzy, principalmente no que se refere aos dados reais.

\section{Referências Bibliográficas}

Aguiar, H., Oliveira, Jr., 1999. Lógica Difusa - Aspectos Práticos e Aplicações; Editora Interciência, 1a Edição.

Barron, J. J., 1993, A Lógica Fuzzy em Foco, Byte No 06, pág. $94-100$.

Doll, H. G., 1949, Introduction to Induction Logging and Application to Well Drilled with Oil Mud: Journal of Petroleum Technology, vol. 1, no. 6, p. 148-162.

Ellis, D.V., 1987, Well Logging for Earth Scientists. Amsterdam, Elsevier. 532p.

Gabriel A. C. Lima; Alexandre C. Vidal; Saul B. Suslick, Estimativa da incerteza na porosidade presente em dados depetrofísica e geofísica de poço. XXVI IBERIAN LATIN AMERICAN CONGRESS ON COMPUTATIONAL METHODS IN ENGINEERING, Guarapari, ES, Brazil, 2005.

Guerra, C. E., "Deconvolução de Perfis de Poços Através do Ajuste de Energia", Belém, Universidade Federal do Pará, Centro de Geociências. 80p. Tese (Mestrado em Geofísica), Curso de Pós-Graduação em Geofísica, Centro de Geociências, 1994.

Gomide, F.A. Campos; Gudwin, Ricardo R., 1994, Modelagem, Controle, Sistemas e Lógica Difusa; SBA Controle \& Automação/ vol.4.

Loupas,T. W. Mcdicken, P. Allan.,1989, An adaptive weighted median filter for speckle suppression in medical ultrasonic images, IEEE Trans. Circuits Syst.

Miranda, V., 1998, Fundamentos de controlo difuso (fuzzy control). Texto básico para a disciplina 'Controlo Difuso e Redes Neurais, do curso de Engenharia Eletrotécnica e de Computadores da FEUP - Faculdade de Engenharia da Universidade do Porto, DEEC - Departamento de Engenharia Electrotécnica e de Computadores. Portugal.

Ortega, N.R.S. 2001, Aplicação da Teoria de Conjuntos Fuzzy a Problemas da Biomedicina. Tese (Doutorado em Física) - Instituto de Física da Universidade de São Paulo. São Paulo, 152 f.

Russo,F., G. Ramponi., 1996, "A Fuzzy Filter for Images Corrupted By Impulse Noise", IEEE Signa I Processing Letters, No . 6 ,pp . 168-170.

Serra, O., 1984, Fundamentals of Well-Log Interpretation. Amsterdam, Elsevier. 432p. 